3 social media sites. We analysed the brand presence and page content on each site and assessed the use and effectiveness of age restrictions.

Results Facebook was the most-used social media site, with an average reach across the observation period ranging from $39 \%$ in males aged 6-14 to $91 \%$ among females aged $15-24$. The average impressions per month varied between 697 million and 2,717 million. YouTube had a similar average reach (41-81\%) while Twitter had a considerably lower usage in the age groups studied. All 5 of the alcohol brands studied maintained a brand website, facebook page and twitter page, while 3 of the 5 also hosted a YouTube channel. Features such as the 'like' button on facebook and the use of competitions and games enable spread of brand engagement through the network.

Age restrictions to alcohol brand content varied across the sites. Facebook users under the age of 18 years were not able to access 'official' alcohol brand pages, although most user-generated content and some brand-generated applications were still accessible. By contrast, YouTube and Twitter did not maintain age-restriction with users of all ages able to view and interact with brand content.

Conclusion Social media sites are heavily used by children and young adults. Their exposure through these sites to alcohol marketing warrants intervention.

\section{PS30 THE JOINT EFFECT OF UNEMPLOYMENT AND CYNICAL HOSTILITY ON ALL CAUSE MORTALITY}

doi:10.1136/jech-2012-201753.129

U Christensen, MK Kriegbaum, NR Rod, MO Osler, RL Lund. Department of Public Health, Section of Social Medicine, University of Copenhagen, Copenhagen, Denmark

Background Unemployment as well as hostility has been associated with mortality and morbidity. Hostility and socioeconomic position, including unemployment is highly associated. One of the hypothetical models on the relation between hostility, health and life context, states that hostility moderates the relationship between health problems and stressful conditions in the environment, such as unemployment. The aim of this study is to analyze the joint effect of labor market exclusion and hostility on all cause mortality.

Methods This study is based on The Danish Longitudinal Study on work, Unemployment and Health, a survey carried out in the Spring 2000 among a random sample of 40 and 50 year old men and women and an oversampled group of previously unemployed individuals. The survey included self-reported measures on employment, education, age and hostility, measured by the eight-item Cynical Distrust Scale.

The exposure variable was: 1) employed-not hostile ; 2) employed - hostile; 3) unemployed-not hostile and 4) unemployed - hostile. Outcome was defined as all-cause mortality. We used Cox's proportional hazard regression model, with age as the underlying time scale and with entry time January 1st 2000. All individuals who reported not working due to illness at the time of the survey were excluded in the analyses. The joint effect of unemployment and hostility was assessed as departure from multiplicativity.

Results Employed men and women who were hostile did not have an increased mortality risk. Unemployed men had an increased risk of mortality even when they were not hostile $\mathrm{HR}=2.30$ (95\% CI, 1.27-4.16) and the joint effect of unemployment and hostility was higher than what would have been expected from their separate effects, HR=2.57 (95\% CI, 1.50-4.42). Unemployed women did not have a significantly increased mortality risk if they were not hostile $\mathrm{HR}=1.35$ (95\% CI, 0.73-2.50), however, the joint effect of unemployment and hostility was higher than what would have been expected from their separate effects, $\mathrm{HR}=2.23$ (95\% CI, 1.17-4.24).

Conclusion The joint effect of unemployment and hostility is a novel finding, indicating that the health damaging consequences of unemployment are accentuated by hostility.

\section{PS31 PATTERNS OF PARTNERSHIP SMOKING DURING PREGNANCY}

doi:10.1136/jech-2012-201753.130

SL Prady, H Graham, KE Pickett. Department of Health Sciences, University of York York, UK

Background Partner's smoking status is an important predictor of maternal smoking in pregnancy; however there are few UK-wide surveys which look at these effects. We investigated partnership smoking, quitting and cutting down during pregnancy in the UK's Millennium Cohort Study.

Methods We used multinomial logistic and linear regression to investigate retrospective self-reported smoking status and reductions in cigarettes smoked of mothers and their partners. All analyses were weighted for the complex sampling design and non-response, both unadjusted and adjusted for socio-demographic status.

Results Seventy-six percent of women had respondent partners, $22 \%$ were couples who both smoked around the time of the pregnancy (unweighted $\mathrm{N}=2,954$ ). Of these smoking couples, $37 \%$ of women quit during their pregnancy, $41 \%$ cut down, and $23 \%$ smoked the same amount (persistent smokers), respective percentages for partners were $16 \%, 25 \%$ and $56 \%$. Compared to women who quit, women who cut down were more likely to have a partner who was a persistent smoker (RRR 7.6, 95\% CI 4.9 to 11.7 ), or a cut-down smoker (5.7, 3.8 to 8.5). Compared to persistent smokers, those who cut down were slightly heavier smokers (women mean difference 1.1 cigarettes/day, partners 2.0). Women who cut down reduced average consumption to 6.3 a day (mean reduction $9.3,95 \%$ CI 9.0 to 9.8 ) and partners to 10.1 (8.7, 8.1 to 9.3). There was no difference in the effect of partners' persistent smoking on the risk of women cutting down or persistent smoking $(\mathrm{P}=0.38)$, but partner cutting down was a significantly larger predictor of women cutting down compared to women continuing to smoke ( $\mathrm{P}=0.02$ ). After reduction, women whose partners cut down smoked an average of 2.2 more cigarettes more than those whose partners quit $(\mathrm{P}=0.043)$. There was no evidence of variation in reduction for those who had partners who quit and those who were persistent smokers $(d=0.87$, $\mathrm{P}=0.42$ ). Results were robust to adjustment for socio-demographics; fully adjusted results will be presented.

Conclusion Most women cut down and this was associated with partner cutdown, however this strategy was limited as partners were more likely to be persistent smokers. Significant reductions in the maternal number smoked were achieved, but the influence of partner smoking (whether persistent or cut down) may limit reduction. Cutting down as a strategy has questionable impact on inutero exposure and may put at infants at risk for environmental tobacco smoke exposure postpartum. For maximum effect, partner and targeted couples interventions are warranted.

\section{PS32 BREAST CANCER SCREENING UPTAKE IN DIFFERENT ETHNIC GROUPS IN LONDON}

doi:10.1136/jech-2012-201753.131

${ }^{1} \mathrm{RH}$ Jack, ${ }^{1} \mathrm{H}$ Møller, ${ }^{2} \mathrm{~T}$ Robson, ${ }^{1} \mathrm{EA}$ Davies. 'Thames Cancer Registry, King's College London, London, UK; '2London Quality Assurance Reference Centre, London, UK

Background Breast screening uptake is lower in London than other areas of England. Knowledge of cancer screening programmes varies between ethnic groups, and previous studies have shown variation in uptake between broad ethnic groups. This study aimed to determine whether breast cancer screening uptake varies between 16 ethnic groups in London.

Methods Information on women resident in London who were sent a breast cancer screening invitation between 31/03/2006 and $31 / 12 / 2009$ was obtained from the London Quality Assurance 